\title{
Hybrid Wireless Communication using Optical and RF Communication and Industrial Application
}

\author{
Dawei Cai \\ Iwate Prefectural University \\ Iwate, Japan 020-0193
}

\begin{abstract}
This paper presents a new hybrid wireless communication structure that use both optical and RF communication to avoid traffic collision problem happened in normal wireless communication. The new communication protocol can be used to create a wireless communication with large number nodes. According to strictly scheduled wireless transmission, each wireless node transmit packets in a unique time slot, the wireless collision could be completely avoided. To show its performance and possible application, we implemented the wireless communication to DPS that is often used in factories. The result of the experiments for DPS illustrated that the new hybrid communication could provide good performance on both real-time property and reliability, besides, the DPS could achieve the similar performance of typical wire system. With more improvement on the hybrid system implementation, the new hybrid communication system may be expected to be widely applied in industrial field.
\end{abstract}

\section{General Terms}

System Development

\section{Keywords}

Optical communication, RF communication, Wireless communication, Traffic Collision

\section{INTRODUCTION}

Wireless communication has been used in industrial applications for more than 30 years. Among the first applications where wireless was used was in wireless control of Automated Guided Vehicles (AGV) and cranes in warehouses where proprietary radios where used to achieve flexible control of the moving devices. During the last 10 years, standardized radio technologies like Wireless LAN / WLAN (IEEE 802.11), IEEE 802.15.4 and Bluetooth technology (IEEE802.15.1) have become the dominating technologies for industrial applications. The main advantages for using a wireless solution in industrial applications are the following:

(1) Greater mobility and possibility to move devices and connect to smartphones and tablets freely without constraining cables.

(2) Eliminate expensive and maintenance heavy transmission media such as flexible cables, swivels, etc.
(3) Bypassing long distances and areas where cables cannot physically fit.

(4) Fast and easy installation and commissioning.

(5) High flexibility if there is a need to modify an installation

(6) Increased personnel safety by not having to be physically close to a device during configuration and/or maintenance.

(7) Flexible Human Interface Devices (HID).

(8) Easy integration of devices into the network.

Industrial plants consist of multiple devices interconnected in different ways. These varied types of devices consist of the following: ? Simple data collection units (I/Os) without built-in intelligence. ? Intelligent devices such as sensors with built-in intelligence, single-loop controllers or programmable controllers. ? Supervisory systems used as Human Machine Interface (HMI), data logging and supervisory control.

All these types of devices are interconnected using different communication protocols and media types that in some cases can be replaced by wireless technologies to achieve the above listed advantages.

Recently, Wireless networks are increasing in popularity. Particularly, wireless communication technology and solution, such as Wi-Fi, WLAN, Bluetooth, etc. are being used widely in many cases to provide a ubiquitous network service. Base on this background, wireless technology is also expected to be used in industrial field to provide network with more flexibility, and to boost the efficiency of manufacture. Besides, sensor networks like ZigBee are developing rapidly, used in civil and industrial field to monitor and control environment, energy use, etc. [1], [2]. [3], [4] However, there are some restrictions of extant wireless network make it difficult to be implied in many cases of industrial field. For example, in modern factory, every industrial product contains hundreds of parts, to control the procedure of assembling all the parts, each part would need a communication node to receive command from the control center and to send its state back. This kind of network could be easily constructed by communication cable. Yet, when a wireless network contains that many nodes, it will be difficult to guarantee the real-time property and reliability of communication from wireless collision. Although large portion of work has been made to optimize the collision avoidance algorithm, collision rate still increases exponentially when the number of wireless nodes increases. [5], [6], [7]

To provide a wireless network which is able to guarantee both high real-time property and reliability when the number of nodes in a network is quite large, a hybrid wireless communication 
(HWC) combined with optical and RF communication is proposed. which can avoid packet traffic collision that often happen in wireless communication. And it is also applied in a digital picking system (DPS) to prove the usefulness of the proposal. DPS is a system widely being used in factories to help workers with pick/put operation. Currently, DPS product use special communication cables to construct the communication network. Because it consumes too much time to build and make changes to DPS, the efficiency of production is affected. So if HWC could be used instead of communication cables, the flexibility of DPS will be greatly improved, thus the efficiency of production could be increased.

In the paper, the structure of the HWC and explain the communication protocol is introduced in detail. In order to confirm the validity of the communication structure, we implement the hybrid communication in a DSP system, and carried out communication experiment. In Section 3, the implementation of a DPS with the proposed wireless communication is described. Finally, a summary on the hybrid communication system is given.

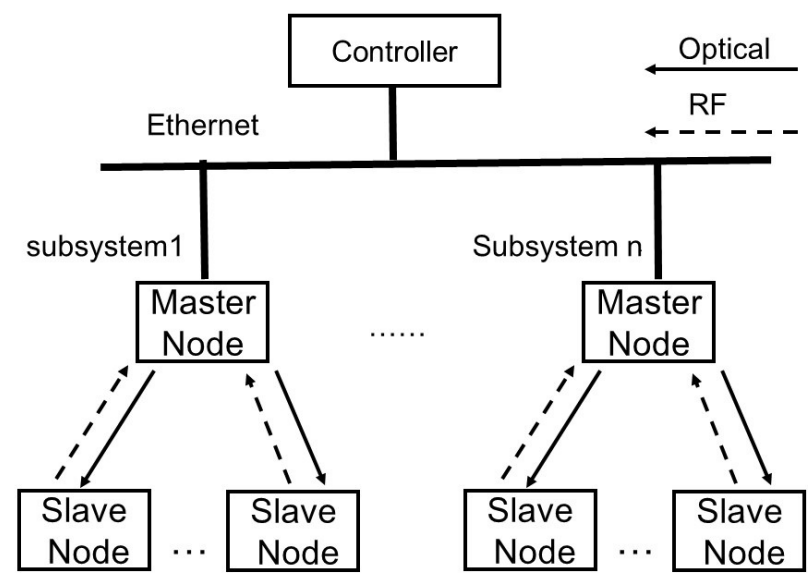

Fig. 1. System topology of the hybrid wireless communication

\section{GENERAL NETWORK STRUCTURE}

The hybrid communication system proposed is constructed with a controller and plural subsystems which is shaped in a star-topology by one master device and plural slave devices. As shown in Fig 1 . each subsystem has its own space area which is not overlapped with other space of other subsystems. To forbid RF traffic collisions between subsystems, each subsystem will be allocated a unique RF channel to transmit RF packets. To forbid optical communication traffic collision between subsystems, the optical signal from the master of the subsystem will only cover the space of its own subsystem. In a subsystem, the master device may communicate with more than several hundred slave devices. Also the master device make link with upper controller with wire link to conform a larger communication system. The controller can link to Ethernet if necessary. In a typical wireless network of this size, it's necessary to prepare an effect collision avoidance algorithm, so that each device would be able to transmit wireless packet with a high realtime property. If there was no constraint on the communication, each slave could send packet with RF in a random time, and wireless collision could not be completely avoided. This collision problem will destroy the real-time property and reliability of the communication network. In our paper, in order to avoid such traffic collision, we use optical signal to schedule the transmission time of each slave device, and create a unique slot for each slave device. Therefore, there are not more than two slave devices can send the packet at the same time. In our system, the traffic collision will be avoided completely. There are two reasons to choose optical signal rather than RF to transmit schedule message. 1). optical signal can provide a much better BER than RF, which could decrease the error rate in reception of schedule message. 2). optical reception consumes much less power than RF, which could extend the sustainability of a slave device when the slave device is powered by battery.

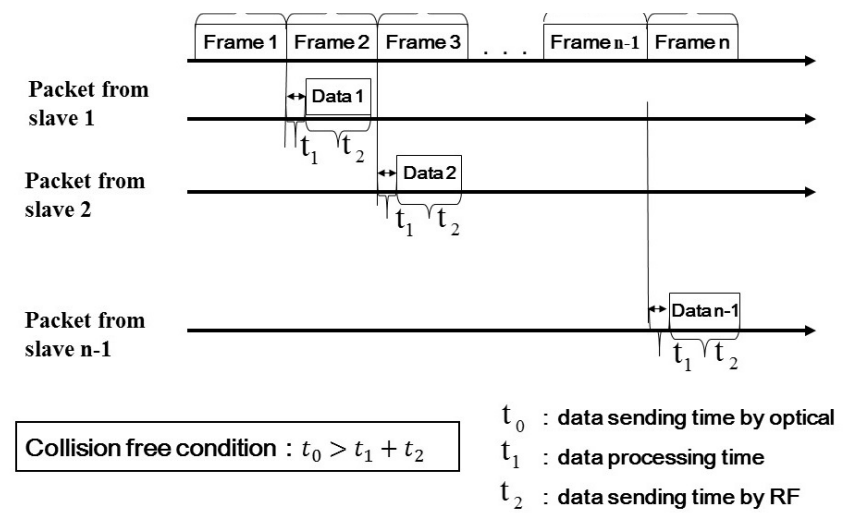

Fig. 2. The mechanism of Wireless collision avoidance

The schedule of wireless transmission is not complex. Each slave device has a unique ID to identify the time slot. During the time slot, the slave device can transmit packet by the RF channel specified by the master device. The optical data sent by master device contains a beginning segment and plural equal length frames. Each frame contains data from master device to a particular slave device which is identified by its device ID. Each slave device determines its RF transmission time base on the optical data flow as shown in Fig 2 As shown in Fig 2, the traffic collision will not happen, if the collision free condition is satisfied. Here, $t_{0}$ is optical frame time in one slot, $t_{1}$ is the time for preparing the sending data in slave device, and $t_{2}$ is the RF sending time of one packet from the slave. It's easy to understand that $t_{1}+t_{2}<t_{0}$ should be guaranteed to avoid any traffic collision. Each frame contains data of the same length, so the time of a frame transmission is identical. A slave will keep receiving infrared data, and count the number of frames received. When the number equals the ID of a slave device, the slave device begins to prepare its data for a coming RF transmission. The slave device processes the frame received in a time $t_{1}$ and begins to send a fixed length RF packet within a fixed time $t_{2}$.

Because optical signal frames contain no schedule message for slaves, the continuity of frame flow is crucial for precisely schedule control of RF transmission. If a frame is missed, two slaves will select the same time slot to transmit RF data, and RF collision will happen. To completely avoid RF collision, we introduced two approaches to guarantee that each slave device could be able to detect error or miss of optical signal frame reception. 
(1) Each slave detects the miss of optical frame by checking the reception time of each optical frame. Each frame has the same length, so in any given optical transmission speed, the transmission time of each frame is also the same. After reception of the beginning of an optical packet, each slave could be able to calculate the reception time of each frame of the packet. So a miss reception of a frame could be detected for the miss receiving in a calculated time. Theoretically, a slave could time precisely for each frame, however, when there are large quantity of frames in each packet, it will be difficult and expensive for a slave device to do so. Hence, instead of timing from the beginning of the optical packet, each slave only time the next frame it will receive, and adjust its timer when it finishes the reception. In this way, slave could easily detect any miss reception of optical frame regardless the number of frames.

(2) To increase the reliability of optical reception, hamming code $(16,11)$ is implemented in each frame for error detection and error correction. The basic hamming code is chosen because it is easy to implement and requires little computation, which is suitable to a cheap slave device with restricted processing ability. Also slaves would check reception time of each frame to insure no frame is missed.

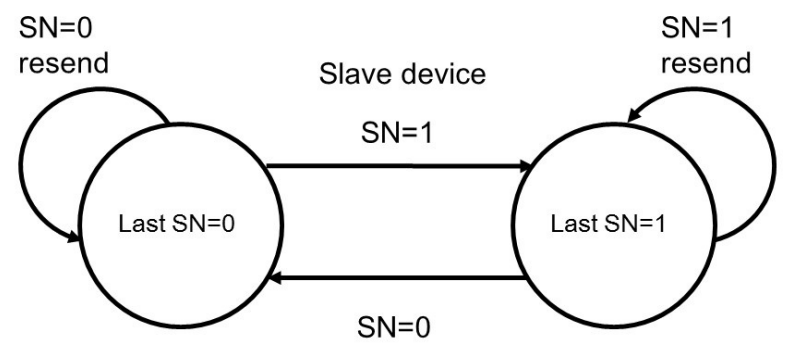

Fig. 3. Mechnism of Serial Number

The optical signal packet does not only schedule the transmission of each slave device, but it also contains data from master device to each slave device. To guarantee that the each slave device receives optical signal data sequentially, a serial number is imported. The serial number is set to one bit to reduce the consumption of payload. The one bit serial number works as shown in Fig 3 A slave device will resend a packet until the serial number changed, and a master will change the serial number when the optical data transmission is confirmed. By this way the continuity of optical data reception could be guaranteed.

\section{HYBRID COMMUNICATION PROTOCOL}

According to the guideline of protocol layering of OSI reference model, a hierarchical protocol structure similar to the OSI reference model was adopted in our hybrid communication as shown in Fig 4 In our hybrid communication structure, the optical media is used for creating downlink that sends data from master device to slave device, and the RF media for creating uplink that transmits data from slave to master. In both master and slave, there are application layer, transport layer, network layer, data link layer and physical layer for data transmitting and processing. As shown in Fig 4 the down linking by optical media and up linking by RF media can be used at the same time, and no need to switch the channel and etc. For the optical can provide a good communication with very low BER (bit error rate) than RF and has less influences from environment, especially in an industrial environment, so stable communication may be expected for industrial application.

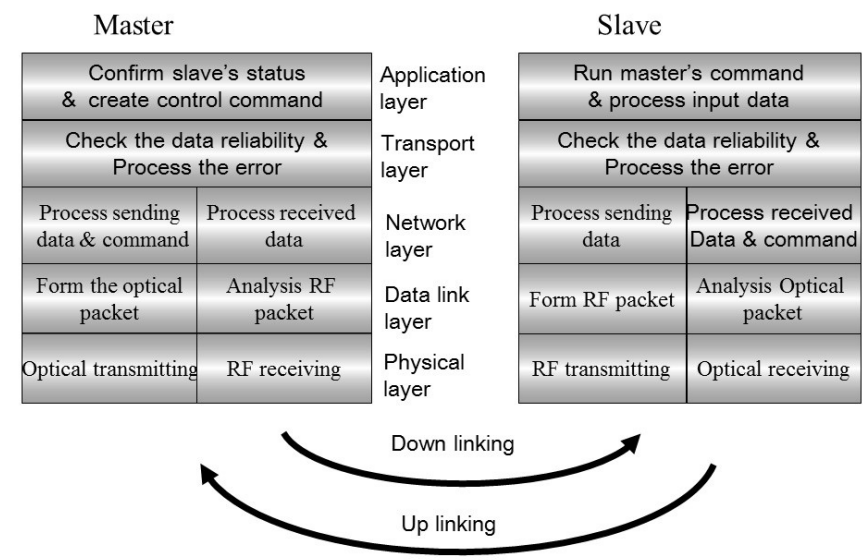

Fig. 4. Hierarchicl protocol of hybrid communication

Fig 5 and Fig 6 illustrate the protocol stack of the hybrid communication for optical and RF. In down linking by optical, 5 bits are attached to the upper data for code correction while transmitting from master device to slave devices. The slave device will check the correction by using the 5 bits, when the slave device receives the packet. In network layer, master ID and slave ID are attached for data flow control.

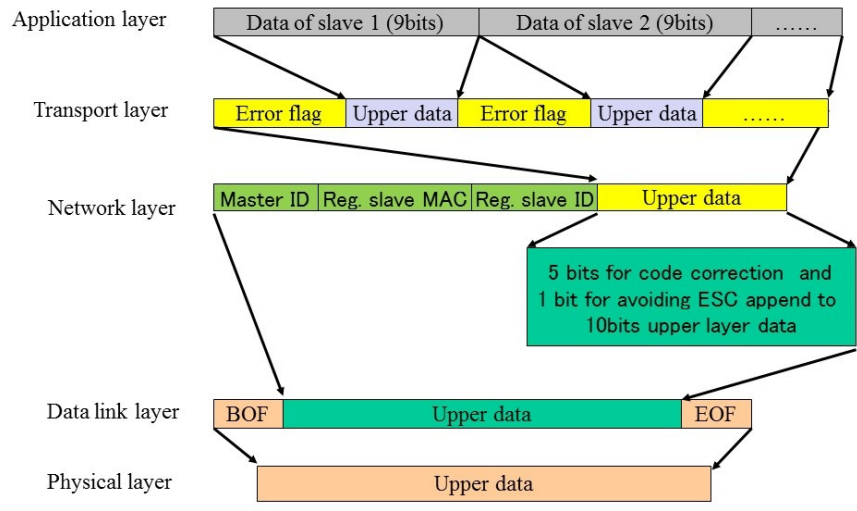

Fig. 5. Optical communication protocol stack

\section{THE IMPLEMENTATION OF PROTOCOL}

To confirm the real-time property and reliability of HWC protocol, we implemented the hybrid communication protocol to a DPS that is often used in assembly factory. DPS device is a system usually being used in assembly lines to help workers with pick/put operation. With hundreds of terminals install on each part shelf, location and amounts of pick/put operations could be controlled electronically, paper work is eliminated and operation error could 


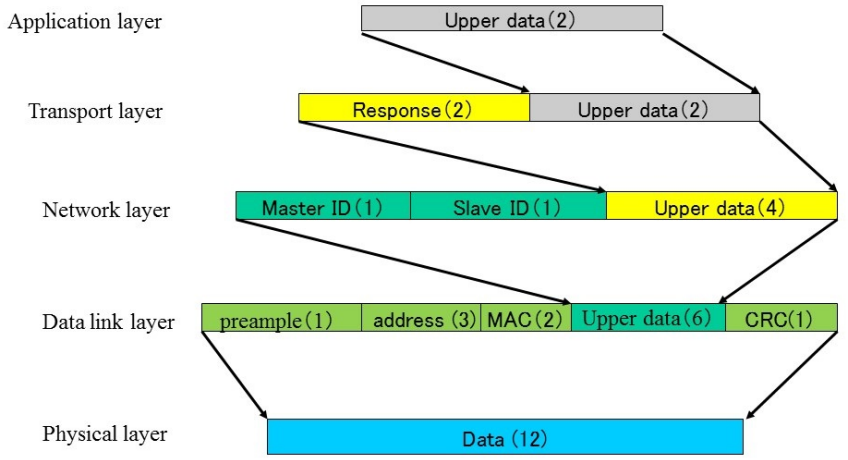

Fig. 6. RF communication protocol stack

be decreased. Because workers operate under the direction of DPS in high speed, the real-time performance is strongly expected. Typically, a DPS is constructed base on the AS-i standard. The standard can provide a network with a performance in Table 1.

\subsection{DPS HARDWARE}

Fig 7 illustrates the main hardware structure of the master device and slave devices. As shown in Fig7 the master device is composed with an ARM726 based control module, an optical transmitting module and a RF receiving module. The slave device is composed with a MSP430 MPU based control module, an optical receiving module, a RF transmitting module, and the operation interface (LED display and input button). The master of HWC is constructed with STMP3600 which is implemented with real time OS ThreadX. A real time OS based hardware is used because the real-time property of the master is important to collision avoidance and the real-time property of the whole network. Slaves are constructed with MSP430 which has very low power consumption, so a slave could be used for a long period without changing battery. Each slave device contains 2 LEDs to show its state, 27 segment displays to show 2 digit numbers which indicate the operation that should be done.

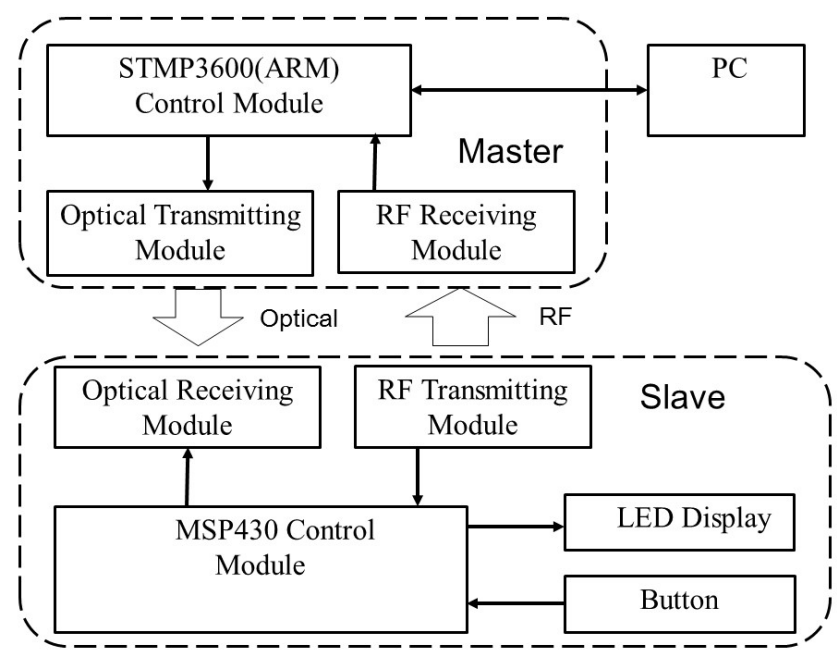

Fig. 7. Hardware of master and slave
Table 1. The performance of DPS and AS-i

\begin{tabular}{|l|l|l|l|l|}
\hline & $\begin{array}{l}\text { Max number } \\
\text { of slaves }\end{array}$ & $\begin{array}{l}\text { Input } \\
\text { ports }\end{array}$ & $\begin{array}{l}\text { Output } \\
\text { ports }\end{array}$ & $\begin{array}{l}\text { Cycle times } \\
(m s)\end{array}$ \\
\hline AS-i & 62 & 248 & 186 & 10 \\
\hline HWC & 128 & 128 & 2028 & 23 \\
\hline
\end{tabular}

Fig 8 shows the PCB module of the master device and slave devices.
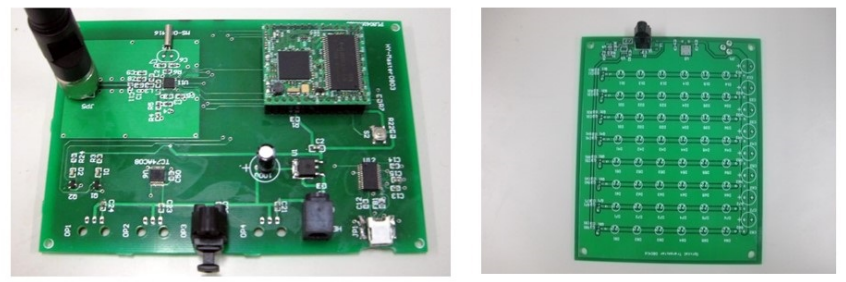

Master Control Module Optical Transmitter

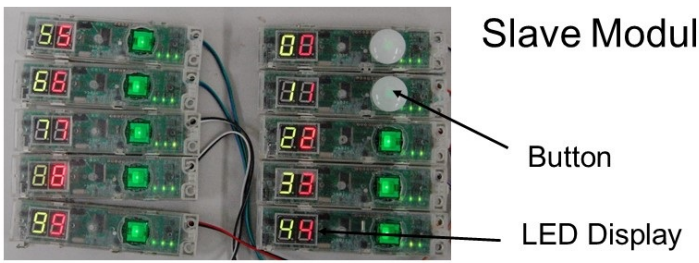

Fig. 8. The master device and slave devices

For physical layer of optical communication, we use infrared chip which support a $115 \mathrm{Kbps}$ IrDA physical specification. For physical layer of RF, NRF24L01 that support $2.4 G H z 2 M b p s$ wireless communication was selected. Each RF packet contains 12 bytes, the transmission speed is $2 \mathrm{Mbps}$, and so the transmission time is $48 \mu \mathrm{s}$. Each IR frame contains 2 bytes, the transmission speed is $115 \mathrm{Kbps}$, and so the transmission time is $156 \mu \mathrm{s}$. The transmission time of optical frame is longer than the transmission time of RF packet, so the RF collision could be avoided. A master of HWC can connect up to 128 slave devices as DPS terminals. An optical packet sent by the master contains 260 bytes, the transmission time is $23 \mathrm{~ms}$. Each slave device has 16 output ports and 1 input ports. Table 1 shows the performance of DPS with HWC along with the performance of DPS under the AS-I standard.

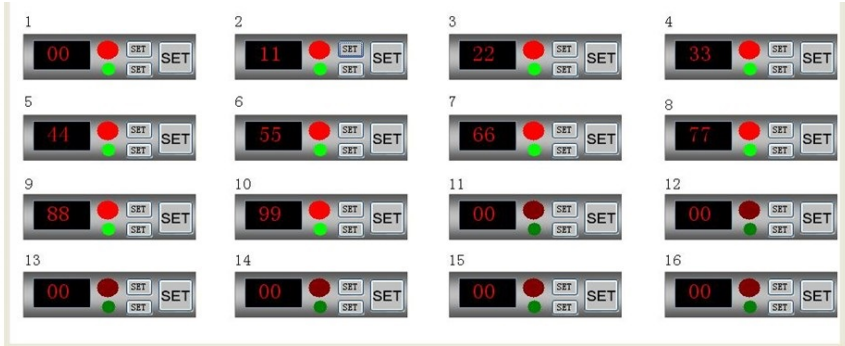

Fig. 9. The window of the control application 
AS-i and the HWC have different maximum number of slaves as shown in Table1. But the average cycle time of every slave in AS-i is $0.16 m s$ which is similar to the average cycle time of every slave in the HWC which is $0.18 \mathrm{~ms}$. So the DPS with HWC could achieve the similar real-time property of a DPS under AS-i standard. The master device is connected to a PC with RS232, and there is an application which can control the slave devices. In the application, we can get the button action information and set the display information of the LEDs through the master device in real time. Fig 9 shows the application windows. In the windows, we can control 32 slave device.

\subsection{COMMANDS FOR DPS CONTROL}

In order to control slave devices of DPS, a set of commands and responses are defined as shown in Table 2 and Table 3 . The commands sent to slave from master are specified in Table 2 . The command is contained in a frame, and is fixed 9 bits in length. With the commands, we can set up the slave device and get the input action from the slave device. The responses of the commands from slave devices are specified in Table 3. Because the responses are sent from slaves by RF, the response is fixed eight bytes in length.

Table 2. Command sent by Master ( The numbers in parentheses indicate the number of digits of the bits)

\begin{tabular}{|l|l|}
\hline CMD Name & CMD format \\
\hline Write DIGIT & 0 digit1(4) digit2(4) \\
\hline Set slave ID & 10 ID(7) \\
\hline Command & 1111 unuse(2) error(1) sleep(1) wake(1) \\
\hline Read slave state & $\begin{array}{l}110 \text { input(1) Delete(1) } \\
\text { Digit1(1) Digiti2(1) LED1(1) LED2(1) }\end{array}$ \\
\hline Set LED & 1110 unuse(1) LED1(1) LED2(1) \\
\hline
\end{tabular}

Table 3. CMD Responses from slave (The numbers in parentheses indicate the number of digits of the bytes)

\begin{tabular}{|l|l|}
\hline R. Write DIGIT & $\begin{array}{l}\text { MAC(2) Master ID(1) Slave ID(1) } \\
\text { data received(2) old DIgits(1) unuse(1) }\end{array}$ \\
\hline R. Set slave ID & $\begin{array}{l}\text { MAC(2) Master ID(1) Slave ID(1) } \\
\text { data received(2) old ID(1) unuse(1) }\end{array}$ \\
\hline R. Command & $\begin{array}{l}\text { MAC(2) Master ID(1) Slave ID(1) } \\
\text { data received(2) old state(1) unuse(1) }\end{array}$ \\
\hline R. Read lave state & $\begin{array}{l}\text { MAC(2) Master ID(1) Slave ID(1) } \\
\text { data received(2) input LED1,2(1) digit1,2(1) }\end{array}$ \\
\hline R. Set LED & $\begin{array}{l}\text { MAC(2) Master ID(1) Slave ID(1) } \\
\text { data received(2) old LED(1) unuse(1) }\end{array}$ \\
\hline
\end{tabular}

\section{CONCLUSIONS}

Wireless technology and application are expected to be used in various industrial fields to improve the flexibility and efficiency of manufacturing. Because there is a very high request for the real-time property and reliability, the extant wireless network protocols are difficult to be directly used in industrial field. In wireless communication, traffic collision issue, when the scale of network aggrandizes. To solve the problem, we proposed a hybrid wireless communication with optical and RF to address this challenge. The HWC could guarantee real-time property and reliability of communication from wireless communication traffic collision. To confirm the performance of HWC, a DPS with HWC was implemented. The result of performance experiments of DPS with HWC shows that the proposal could provide a high real-time property and high reliability network for industrial field. it is shown that the hybrid communication presented is useful for system which transit small amount information in real time. We will continue to improve the hybrid protocol and try to popularize it to industrial field to improve the efficiency manufacture in future.

\section{REFERENCES}

[1] A Andreas, C T Mattias - Sweden, ZigBee: a suitable base for embedded wireless development? , Chamers University of Technology, 2005

[2] Koubaa, C A. Alves, C M. Tovar, GTS allocation analysis in IEEE 802.15.4 for real-time wireless sensor networks, Parallel and Distributed Processing Symposium, 2006

[3] As-Interface, www.as-interface.net

[4] K. Das and P. Havinga, Evaluation of DECT-ULE for Robust Communication in Dense Wireless Sensor Networks, 3rd International Conference on the Internet of Things, IOT 2012. IEEE Communications Society, 2012, pp. 193-190.

[5] L. L. Bello and O. Mirabella, Communication techniques and architectures for Bluetooth networks in industrial scenarios, Emerging Technologies and Factory Automation, 2005. ETFA 2005. 10th IEEE Conference on, vol. 1, 2005

[6] A. Willig, K. Matheus, and A. Wolisz, Wireless Technology in Industrial Networks, Proceedings of the IEEE, vol. 93, no. 6, pp. 1130-1151, 2005.

[7] D. Brevi, D. Mazzocchi, R. Scopigno, A. Bonivento, R. Calcagno, and F. Rusina, A Methodology for the Analysis of 802.11a Links in Industrial Environments, Proc. 2006 IEEE International Workshop on Factory Communication Systems (WFCS), July 2006. 